\title{
Adquisición tardía de la primera lengua: experiencia escolar de niñas y niños Sordos de una Institución Educativa pública en la ciudad de Popayán (Cauca) ${ }^{1}$
}

Later acquisition of first language: educative experience of Deaf girls and boys at a Public School in Popayan (Cauca)

Aquisição posterior da língua materna: experiência escolar de crianças surdas de uma instituição estatal de ensino elemental na Cidade de Popayán, estado do Cauca, Colômbia.

\section{Luz del Sol Vesga Parra ${ }^{2}$ \\ Danelly Lasso Quintero ${ }^{3}$ Mónica Marcela Tobar Londoño ${ }^{4}$}

${ }^{1}$ Este artículo es el resultado de la investigación "Experiencias escolares en la adquisición de la Lengua de Señas por niñas y niños Sordos en el Aula Básica para Sordos de una Institución Educativa pública en la ciudad de Popayán (Cauca-Colombia), durante el año 2014- 2016. Las autoras desean dar expreso crédito y mención a la Corporación Universitaria Autónoma del Cauca (Popayán), por los espacios y tiempos facilitados para llevar a cabo esta investigación, así como a la Institución Educativa la Pamba (Popayán).

${ }^{2}$ Estudiante de Doctorado en Ciencias de la Educación (Universidad del Cauca - RUDECOLOMBIA), Magíster en Educación (Universidad del Cauca). Licenciada en Tecnología e Informática (Universidad Católica, Manizales). Docente Investigadora de la Facultad de Educación de la Corporación Universitaria Autónoma del Cauca. Integrante del Grupo de Investigación GICHIS y Coordinadora del Semillero de Investigación SEFAC de la Corporación Universitaria Autónoma del Cauca. Docente en el aula básica para Sordos de la Institución Educativa la Pamba, sol.vesga@uniautonoma.edu.co

${ }^{3}$ Tesista de la Licenciatura en Educación Preescolar (Corporación Universitaria Autónoma del Cauca), Integrante del Semillero de Investigación SEFAC de la Corporación Universitaria Autónoma del Cauca, danellylq@hotmail.com

${ }^{4}$ Tesista de la Licenciatura en Educación Preescolar (Corporación Universitaria Autónoma del Cauca), Integrante del Semillero de Investigación SEFAC de la Corporación Universitaria Autónoma del Cauca. Contacto:monicat204@hotmail.com 


\section{Resumen}

El artículo presenta el resultado de una investigación que buscó comprender las experiencias vividas por las niñas y los niños Sordos frente al acceso y adquisición tardía de su primera lengua (Lengua de Señas Colombiana), de una Institución Educativa pública de Popayán que implementa desde hace 4 años un método de educación bilingüe y bicultural para la población Sorda. La investigación fue cualitativa, basada en los principios del método hermenéutico fenomenológico, se utilizaron algunas técnicas de recolección como la observación participante, el análisis documental y la entrevista a profundidad. Se indagó desde las realidades de los sujetos de la investigación sus diversas experiencias antes y después de adquirir su primera lengua, logrando comprender vivencias personales, familiares y escolares que allí se gestaron. Los hallazgos más importantes fueron las transformaciones de vida por las niñas y niños Sordos al adquirir su lengua natural, por ejemplo se evidenciaron avances en la interacción social, en la expresión de sus ideas y emociones, en su interés por aprender, en su fortalecimiento de identidad y autoestima, en la generación de oportunidades para la participación, en la posibilidad de ser escuchado, entre otros.

Palabras Clave: Escuela, Experiencia, Lengua, Niñez, Sordos.

\section{Abstract}

This paper shows the results of an investigation intended to understand the experiences of Deaf girls and boys about the access and later acquisition of their first language (Colombian Sign Language) at a Public School in Popayan, which has implemented, from 4 years ago, the bilingual and bicultural educational method to Deaf population. This was a qualitative investigation, it was founded on the tenets of the phenomenological and hermeneutics methodology, it were used some technics of collect data like the participative observation, documental analysis and in-deep interview. From realities of Subjects of investigation, it were searched some experiences before and after acquire their first language. The investigation allowed understanding the personal, 
familiar, and school experiences engendered there. The most outstanding findings were related with the life transformations of the girls and boys in the process to acquire their natural language, for example, it was evidenced progress in the social interaction, the expression of their ideas and emotions, their motivation to learn, the strengthening of the identity and self-esteem, the creation of opportunities to participate, the possibility to be heard, etc.

Keywords: School, Experience, Language, Childhood, Deaf people

\section{Resumo}

$\mathrm{O}$ artigo apresenta o resultado de uma pesquisa que tinha como alvo compreender as experiências vividas por crianças surdas em relação ao acesso e aquisição tardia da sua primeira língua, conhecida na Colômbia como Língua de Sinais Colombiana e no Brasil como Língua Brasileira de Sinais - LIBRAS, numa instituição estadual de ensino Elemental na cidade de Popayán. A unidade educativa desenvolveu há quatro anos um método bilíngue e com diversidade cultural para a população surda, focado na melhora da sua qualidade de vida e relacionamento com o seu entorno. A pesquisa foi de tipo qualitativo, baseado no método hermenêutico fenomenológico, além de serem utilizadas algumas técnicas de coleta de dados, tais como a observação-participante, análise documental e entrevista em profundidade. Para isto foram feitos questionamentos, respeito às realidades dos sujeitos alvo de estudo, sobre as suas diversas experiências antes e depois de adquirir a sua primeira língua, permitindo compreender as experiências pessoais, familiares escolares geradas no processo de aprendizado. Os resultados mais relevantes foram: as transformações de vida das crianças surdas no processo de aquisição da sua língua natural, o seu interesse pela aprendizagem, o fortalecimento da sua identidade e autoestima. $\mathrm{O}$ anterior refletiu no progresso de interação social, na expressão das suas ideias e emoções, de forma que se geraram oportunidades de participação social com o dialogo, tendo a possibilidade de ouvir e ser ouvido.

Palavras-chave: escola, experiência, língua, crianças, surdos. 


\section{Introducción}

En la historia de la humanidad se han presentado distintos atropellos sociales, físicos y psicológicos hacia las personas en condición de discapacidad; es así como, en general, han prevalecido conceptos de "normalización" hacia los sujetos, que se han ido materializando en procesos de rehabilitación (Orozco, 2014). Estas miradas han generado atrasos en las poblaciones vulnerables, definidas por el Ministerio de Educación Nacional como: "Personas que, por su naturaleza o por determinadas circunstancias, se encuentran en mayor medida expuestas a la exclusión, la pobreza y los efectos de inequidad y la violencia de todo orden" (MEN, 2005:10), quedando marginadas socialmente y presenciando manifestaciones de exclusión que las aleja de procesos de equidad y justicia social (Bolívar, 2012).

Para el caso de las personas Sordas ${ }^{5}$ no ha sido la excepción, pues las aceptaciones sociales de los oyentes han ido cambiando a lo largo de la historia de acuerdo a la concepción cultural del grupo social al que se pertenece; por ejemplo: en algunas comunidades religiosas asociaban la Sordera con el pecado y, entonces, la personas sordas debían morir en una hoguera (Patiño, Oviedo \& Gerner, 2001); en otras, veían a dichas personas como minusválidas, por lo que había que recluirlas en conventos y sanatorios donde les ofrecieran un cuidado asistencial, pero les quitaban algunos derechos como casarse o tener hijos (Medina, 2005). También eran considerados "bobos" y "retardados", pues la persona Sorda, al tener obstruido el canal auditivo, no lograba adquirir un lenguaje, viéndose afectado gran parte del desarrollo del pensamiento (Sacks, 2003).

¿Cómo lograr que los Sordos adquieran una lengua? Esta pregunta acompañó la educación de los Sordos por mucho tiempo. Desde sus inicios, en 1620, Fray Ponce de León crea los primeros métodos orales,

${ }^{5}$ Patiño, Oviedo y Gerner (2001) plantean "que cuando se haga referencia a aquellos sordos que han aceptado su condición sociológica y lingüística se utilice la palabra sordo/a con mayúscula, en oposición a la mirada puramente audiológica" (p. 7). En este artículo adoptamos esta sugerencia. 
por medio de la dactilología $;$; posteriormente, en Francia (siglo XVIII), se abren las primeras escuelas para Sordos, allí se enseña la lengua de señas y se abren espacios participativos a las comunidades de Sordos; sin embargo, estos avances se vieron afectados por la decisión tomada en el Congreso Internacional sobre la Instrucción de los Sordomudos (Milán-1880) donde se prohíbe el uso de las lenguas de señas en las escuelas para Sordos y se promulga el método oral como el único válido; se expresaba así "la superioridad del habla sobre los signos para incorporar a los sordomudos a la vida social... se declara el método oral puro como el mejor" (Oviedo, 2006:13). Estas leyes estuvieron vigentes hasta 1960 en Europa y 1980 en España.

De esta manera, a nivel mundial se castigó de múltiples maneras a los Sordos para que no utilizaran su lengua de señas y así adquirieran la oralidad por distintos métodos. Esto condujo al silenciamiento de la comunidad Sorda por más de un siglo. Fue a partir de 1960 que se inició una nueva época de visibilización y reconocimiento, no solo de la lengua de señas, sino de la cultura Sorda, esto gracias a protestas y movimientos sociales de muchas personas (Sacks, 2003). Es así como alrededor del mundo se conforman asociaciones que defienden a las comunidades de Sordos. En Colombia, en 1984, se funda FENASCOL (Federación Nacional de Sordos de Colombia), cuyos propósitos son: la defensa de los derechos de las personas sordas, el desarrollo, la enseñanza e investigación de la lengua de señas colombiana, la formación de líderes sordos, entre otros.

Antes de estos movimientos sociales, la educación para los Sordos en Colombia estaba a cargo de instituciones religiosas y de fundaciones; en ellas predominaron los métodos de enseñanza oral (Patiño, Oviedo \& Gerner, 2001). La lengua de señas no era reconocida ni utilizada en los espacios escolares. Una vez se inicia el reconocimiento mundial de la cultura Sorda y su diferencia lingüística, se crea en Colombia, en 1956,

\footnotetext{
6 "Sistema de comunicación que transmite información mediante el uso de los dedos de la mano, la gran mayoría para el recuento numérico o la representación de alfabetos manuales" (González, 2011:10).
} 
el INSOR (Instituto Nacional para Sordos), hoy adscrito al Ministerio de Educación Nacional, instituto que ya cumple 60 años de trabajar por mejorar la educación de los Sordos, desde un enfoque socio-antropológico $^{7}$ de la Sordera, y con el método de educación bilingüe ${ }^{8}$ y bicultural ${ }^{9}$.

Las primeras personas Sordas beneficiadas por el INSOR y FENASCOL fueron las que vivían en las grandes capitales colombianas; por el contrario, en ciudades periféricas ${ }^{10}$ de Colombia, como Popayán, hasta hace apenas unos cuatro años llegaron estos movimientos, frente a los nuevos cambios de la educación para los Sordos. La demora en este proceso generó un atraso educativo y social en la población Sorda de estos lugares apartados, pues al no adquirir una lengua desde temprana edad el desarrollo de sus estructuras y procesos cognitivos y comunicativos fueron limitados, ocasionando una segregación y exclusión en todos los sectores de la sociedad (Cabezas, 2104).

En 2012, se crea en Popayán la primera Aula Básica para Sordos ${ }^{11}$ (en adelante ABS), para ofrecer una educación distinta a los Sordos de la ciudad que habían estado más en rehabilitaciones orales que en

${ }^{7}$ Este enfoque, propone la depatologización de la persona sorda. Considera al sordo miembro de una comunidad lingüística minoritaria y marginada, que posee una lengua propia y características socio-culturales propias (Massone \& Behares, 1990, citados por Rey, 2008).

${ }^{8}$ Este método utiliza la primera lengua (lengua de señas) como mediación para el proceso formativo de las personas Sordas, y como segunda lengua, la lengua mayoritaria de la comunidad oyente que se enseña de manera escrita (Lissi, Svartholm, \& González, 2012).

${ }^{9}$ Se refiere a la formación de personas Sordas bajo dos contextos culturales distintos: el de la cultura Sorda, que es minoritaria, y el de la cultura oyente, que es mayoritaria (Robles, 2012).

${ }^{10}$ Entendidas como zonas limítrofes del país, lejos del centro; también hacen parte ciudades donde emergen clases sociales bajas, carentes de estructuras edificadas, así como sistemas urbanos, donde el desarrollo social y económico es mínimo (Niño, Velasco \& Toro, 2005).

${ }^{11}$ Aulas multigrado (preescolar hasta quinto de primaria) donde enseña un docente usuario de Lengua de Señas Colombiana y un modelo lingüístico (Sordo adulto), se emplea el método bilingüe y bicultural (MEN, 2009). 
procesos de formación. A los estudiantes Sordos que llegaron al aula se les observó la falta de dominio en una lengua y todas las implicaciones que esto tiene en las dimensiones emocional, cognitiva, social y corporal. Han pasado cuatro años y gracias a las experiencias escolares de los niños y los jóvenes Sordos en la adquisición de su primera lengua, estos han logrado reencontrarse y transformarse gozando de un reconocimiento escolar y social. Este hecho fue la motivación central para iniciar la presente investigación, pues para los estudiantes Sordos había pasado ya el periodo crítico para adquirir su primera lengua ${ }^{12}$; no obstante, los sujetos la aprendieron, sin desconocer que este suceso tenga huellas imborrables (Sacks, 2003).

Por lo anterior, nace el interés por la indagación de las experiencias vividas de los estudiantes Sordos en la adquisición de su primera lengua, asumiendo el concepto de experiencia como un acontecimiento particular de cada persona: "la experiencia son cosas que me pasan a mí, sobre mí, son cosas que pasan fuera de mí y me afectan de tal modo que pueden hacer cambiar mi forma de pensar, de sentir, de vivir" (Larrosa, 2006:3). De este modo, surgieron interrogantes como: ¿qué acontecimientos vivieron los estudiantes Sordos en su proceso de adquisición de la lengua? ¿Qué cambios tuvieron en su vida después de aprender la lengua? ¿Qué transformaciones a nivel personal impactaron sus contextos inmediatos? ¿De qué manera se empiezan a configurar como sujetos sociales? Con estas y muchas otras preguntas nos sumergimos en la realidad y la historia de cada uno de los estudiantes Sordos para lograr develar sus experiencias frente a la adquisición de su primera lengua. El resultado de este proceso lo presentamos en este artículo.

La investigación abordada se convierte en el primer referente desde un lugar periférico de Colombia donde, a través de esfuerzos de distintas personas e instituciones, se ha aportado al rompimiento de las brechas de inequidad y la falta de oportunidades para este tipo de poblaciones

12 "Hay aprendizajes, entre los que está el de la primera lengua, sometidos a periodos críticos, lo que quiere decir que, agotado ese periodo, ya no será posible adquirir tal habilidad" (Monsalve \& Núñez, 2016:15). 
vulnerables. Igualmente, los hallazgos de la investigación sugieren reflexiones educativas para los docentes en ejercicio que deseen ampliar su conocimiento frente a caminos significativos para llegar a la tan anhelada educación inclusiva de estudiantes Sordos; solo así se contribuirá a la construcción de una cultura de inclusión social y educativa en los lugares apartados del país, donde a las personas con sordera se les respete y se les garantice sus derechos lingüísticos y culturales (Domínguez, 2009).

\section{Escenario y participantes}

El contexto de la investigación fue una Institución Educativa pública urbano marginal del sector sur oriental de la ciudad de Popayán, donde se atiende una población vulnerable de estratos socioeconómicos o, 1 y 2. En su entorno social, los estudiantes presentan problemáticas sociales como: drogadicción, pandillas, prostitución, delincuencia común, entre otras. En la historia de la institución educativa se había atendido a estudiantes Sordos, pero solo hasta 2012, cuando se crea la primera ABS en la ciudad, se ofrece una educación bilingüe y bicultural, desde un enfoque socio-antropológico de la sordera, siguiendo las directrices del INSOR.

Actualmente, en la Institución hay 460 estudiantes y nueve de ellos se encuentran en situación de sordera. Para estos estudiantes Sordos se han adecuado los espacios escolares y se cuenta con los profesionales de apoyo para que a través de lengua de señas avancen en su proceso formativo. En el caso del ABS, los estudiantes Sordos de básica primaria son guiados por un docente usuario de lengua de señas y un modelo lingüístico (Sordo adulto bachiller y nativo en la Lengua de señas). Los estudiantes Sordos de bachillerato son acompañados por intérpretes de Lengua de señas a Lengua Castellana y un docente usuario de Lengua de Señas Colombiana que les enseña la lengua Castellana de manera escrita como su segunda lengua. El propósito de este modelo de educación para los Sordos es que no solo terminen sus estudios de bachillerato, sino que, como lo hace cualquier persona oyente, los Sordos puedan acceder y terminar estudios de pregrado y posgrado, según sus intereses particulares. 
Las niñas y los niños del ABS tienen una edad que oscila entre los 8 y 15 años; la edad cronológica de los estudiantes es mayor a su edad mental, pues solo hasta después de los 8 años inician su proceso de adquisición de una lengua. Antes de llegar al ABS, la mayoría de los estudiantes Sordos recibió su educación en aulas con oyentes y, en contrajornada, asistían al INALE $^{13}$, donde recibían una educación oralista. Lo anterior influyó en no tener acceso a una lengua desde temprana edad, en carecer de vínculos afectivos y comunicativos con sus padres, en contar con experiencias de fracaso escolar en distintas instituciones. Actualmente, este panorama ha cambiado gracias a las posibilidades que les ha generado el ABS.

\section{Metodología}

Esta investigación es de corte cualitativo, pues se estudiaron los hechos desde su contexto natural, intentando comprender los fenómenos en función de los significados que le dan las personas (Denzin \& Lincoln, 2005). Por consiguiente, las experiencias y los significados que las niñas y niños Sordos dan al adquirir su primera lengua se convierten en el centro del proceso de indagación. Se empleó el método hermenéutico fenomenológico, asumido como un modelo de investigación que se basa en la noción de la experiencia vivida, proporcionando además la base para que los investigadores educativos puedan reflexionar sobre su propia experiencia personal (Ayala, 2008). En este sentido, todo el proceso de recolección de datos y su posterior análisis guardó siempre una estrecha relación con las experiencias, dando origen al esbozo de los resultados que se presentan.

En la investigación se utilizaron las siguientes técnicas de recolección de la información:

La observación participante: se basó en "captar la realidad social y cultural de una sociedad o grupo social determinado, mediante la inclusión del investigador en el colectivo" (García, Ibañez \& Alvira, 2005:14). De esta manera, se vivieron espacios de inmersión con los

${ }^{13}$ Instituto de Audición y Lenguaje. Creado en Popayán en el año 1963. Ofrece una educación a los Sordos desde métodos oralistas. 
estudiantes Sordos con la idea de interactuar con ellos en su contexto escolar natural; los registros de las observaciones se consignaron en diarios de campo.

La investigación documental: esta técnica se define como un conjunto de herramientas, estrategias y recursos que le permiten al investigador construir, de manera indirecta, información y conocimiento sobre un fenómeno de la realidad a estudiar (Martínez, 2004). La fuente de información que se privilegió en esta técnica fue de tipo iconográfico, seleccionando las fotografías que niños y niñas Sordas eligieron como parte de sus experiencias significativas en la adquisición de su primera lengua en el espacio escolar.

Finalmente, la entrevista a profundidad, entendida como encuentro cara a cara entre dos o más sujetos, teniendo diálogos libres sobre un tema, que permitieron lograr cierta libertad para introducir elementos que ayudaron a obtener una información precisa y clara de los participantes sobre el tema de indagación (Batthyány \& Cabrera, 2011). Esta técnica se aplicó cuatro veces a la docente del aula dado que ha sido la persona quién ha dirigido el proceso de la adquisición de la lengua; a los padres de familia de las niñas y los niños Sordos tres veces a cada uno, y de manera grupal a los estudiantes Sordos del aula; allí se logró comparar y profundizar sobre acontecimientos vividos, relacionados con el fenómeno a investigar; el registro de esta información se realizó de forma audiovisual.

Simultáneamente, al aplicar las anteriores técnicas de recolección de información, se implementaron las técnicas de análisis de la información recopilada; para esto se siguieronprocesos de codificación (abierta, axial y selectiva) y la categorización, con sus respectivos memos y diagramas. Finalmente, se realizó la triangulación de las categorías emergentes, con las categorías teóricas y la voz del investigador, para llegar a los resultados (Murcia y Jaramillo, 2009).

\section{Hallazgos}

En este apartado se presentan los hallazgos de la investigación por 
medio de dos categorías centrales: la primera, Experiencias: presos en el silencio, muestra las experiencias de los estudiantes Sordos antes de tener acceso a una primera lengua; este hecho los llevó a seguir una vida silenciada y excluida no solo de su familia sino de los demás entornos; la segunda, Experiencias: descubro la voz en mis manos, referida a las experiencias de los estudiantes Sordos frente a las transformaciones vividas en la adquisición de su primera lengua (Lengua de Señas Colombiana) durante cuatro años de escolaridad en el ABS.

\section{Experiencias: presos en el silencio}

Antes de empezar a desarrollar esta categoría, es importante tener en cuenta que la experiencia es un acontecimiento que no depende de sí mismo; el lugar de la experiencia es cada uno, cada persona hace de la experiencia algo único que repercute en ella, causando así una transformación en sus pensamientos, sentimientos o proyectos. En este sentido, la experiencia es algo subjetivo al ser humano; los cambios que se generan dependen del punto de vista individual, propiciando nuevos acontecimientos y aprendizajes. Es así como la experiencia es un recorrido que nos lleva a generar cambios, pues lo que ocurre a nuestro alrededor afecta algún aspecto de la vida (Larrosa, 2006). Por ende, los estudiantes Sordos del ABS han tenido experiencias que han reconfigurado sus vidas. En tal sentido, en el desarrollo de la presente categoría "Presos en el silencio" se exponen las experiencias que los estudiantes Sordos vivieron antes de adquirir y utilizar su primera lengua.

Para comenzar, es preciso abordar las experiencias educativas de los estudiantes Sordos antes de llegar al ABS; estas habían estado centradas en dos instituciones educativas, una de ellas privada, donde se utilizaba un método oralista. Al hablar de ella, los estudiantes mencionan recuerdos y experiencias dolorosas mediante expresiones como: "me aburría mucho... había que repetir y repetir muchas veces lo mismo" (T:EP/Re:V/P:ES1/G:F/R:16) ${ }^{14}$; "me obligaban a hablar pero no

${ }^{14}$ Estos códigos pertenecen al procesamiento y análisis delos relatos recopilados 
podía hacerlo" (T:EP/Re:V/P:ES2/G:M/R:11); "me regañaban todo el tiempo por no poder hablar" (T:EP/Re:V/P:ES3/G:F/R:18). La otra era una institución pública, donde el estudiante Sordo estaba integrado con estudiantes oyentes; este ambiente originó frustraciones y fracasos escolares por falta de compartir una misma lengua con sus pares y docentes: "veía que la profesora me gritaba, pero yo no le entendía nada" (T:EP/Re:V/P:ES4/G:M/R:5); "nunca tuve amigos en esa escuela” (T:EP/Re:V/P:ES5/G:F/R:22); "yo imitaba a los demás pero no entendía lo que hacía” ( $T: E P / R e: V / P: E S 1 / G: F / R: 14)$. Estas experiencias de fracaso escolar sembraron en ellos sentimientos de inseguridad, miedo y apatía frente a los procesos escolares.

Fueron muchas las experiencias dolorosas que vivenciaron los estudiantes Sordos en esas instituciones no pensadas para ellos; eran como sombras en aulas de clase, sin voz, sin participación, ante unos docentes que se tornaban desinteresados, distantes, con manifestaciones de lástima y con un total desconocimiento de sus propias necesidades lingüísticas; todo esto desencadenó en vivencias amargas, de dolor y sufrimiento por parte de los estudiantes, que solo recuerdan lo mal que la pasaron en aquellos recintos. Estas experiencias develan el fracaso del oralismo (Sacks, 2003), pues: ¿cómo hacer reproducir un sonido a alguien que jamás lo ha escuchado? ¿Cómo generar procesos de participación cuando no hay mediación comunicativa? Por esta razón, la lengua de señas, enseñada desde tempranas edades, permite desarrollar pensamiento y procesos comunicativos efectivos (Patiño, Oviedo y Gerner, 2001).

Sin acceso a una lengua, los estudiantes Sordos no alcanzan a desarrollar ninguna competencia lingüística ${ }^{15}$, es decir, no tenían oralidad ni usaban la Lengua de Señas Colombiana (LSC), como tampoco el castellano escrito, como lo afirman los relatos: "no entendía cuando me hablaban y yo tampoco podía hablar" (T:EP/Re:V/P:ES2/G:M/R:9);

en la investigación, así: T: Entrevista a Profundidad; Re: Registro; V: video; P: participante; E: estudiante Sordo; G: Género; F: Femenino; R: Relato.

${ }^{15}$ Como competencia lingüística se entiende, según Marines, Heredia, Solis y Mena (2014) la "manera como los seres humanos se apropian del lenguaje y lo emplean para comunicarse" (p. 12). 
"en los colegios donde estaba nadie sabía lengua de señas" (T:EP/ Re:V/P:ES3/G:F/R:16); "no entendía tampoco las palabras escritas" (T:EP/Re:V/P:ES4/G:M/E/R:3). En este sentido, las experiencias de los Sordos, sin acceso a ninguna lengua, resultarían, según Lissi, Sartholm y González (2012), en personas semilingües, es decir, que suelen ser medianamente competentes en una lengua (ya sea oral o de señas), y esto implica una mayor construcción de barreras para la inclusión educativa y social de las personas Sordas.

Así pues, al no contar con una competencia lingüística, los estudiantes Sordos pierden la posibilidad de interactuar con su familia y con su entorno; esto genera vacíos en construcciones de conceptos básicos iniciales para comienzos de procesos escolares. Una infancia silenciada, sin lengua, sin retroalimentación: "en la casa él se entretenía solo pues no había nadie que se pudiera comunicar con él” (T:EP/Re:A/P:P3/G:F/R:31); “uno quería como leerle cuentos como a sus hermanos pero era algo imposible" (T:EP/Re:A/P:P5/G:F/R:17). Esta experiencia de sus primeros años hace que ellos presenten una edad mental distinta a la cronológica y evidencien vacíos en nociones básicas de las distintas áreas fundamentales del conocimiento, lo que hace más difícil y lento el proceso de aprendizaje.

Por lo anterior, los estudiantes Sordos, al no contar con una mediación lingüística, tampoco lograron acceder a procesos de aprendizaje, por lo que generalmente eran señalados como "bobos" y "retardados", viviendo experiencias escolares de soledad, frustración, impotencia y resignación, como lo manifiestan en los siguientes relatos: "trabajaba mucho pero nada me salía bien" (T:EP/Re:V/P:ES5/G:F/R:2O); "mejor me la pasaba sola, pues nadie quería hacerse conmigo" (T:EP/Re:$V / P: E S 2 / G: M / R: 12)$; "a veces me desesperaba y empezaba a llorar sin saber por qué" (T:EP/Re:V/P:ES3/G:F/R:15). Estas experiencias llevaron a fracturar las relaciones familiares y escolares de los estudiantes Sordos, pues las expectativas “de ser personas normales" eran cada vez menores para ellos y su familia.

Es así como, en los años que los estudiantes Sordos vivieron sin tener una lengua para comunicarse (entre 8 y 12 años), se enfrentaron a 
un entorno hostil, de burlas e incomprensiones, como lo expresan: "recuerdo que todos se burlaban de mí porque hacía las cosas mal” (T:EP/ $R e: V / P: E S 1 / G: F / R: 12) ;$ "A veces me pegaban o me maltrataban sin yo saber por qué" (T:EP/Re:V/P:ES4/G:M/R:7); "no quería volver a ese colegio, me sentía muy mal, nadie me quería” (T:EP/Re:V/P:ES5/G:$F / R: 24)$. Estas experiencias marcaron la vida de los estudiantes y hoy lo recuerdan con desagrado y como una época oscura que no quisieran volver a vivir.

El no acceder a una lengua en su infancia tiene inmensas repercusiones en las vidas de los estudiantes; según Sacks (2003), esta carencia genera otro tipo de falencias en el comportamiento y en el desarrollo mental de las personas y se estaría condenado a estar desconectado del mundo en que se habita. En la misma línea, Cabezas (2014) plantea que este hecho impide desarrollar habilidades del lenguaje como: expresar, comunicar, imaginar, crear, recrear, construir pensamiento y conocer el mundo, entre otros.

Otro aspecto importante que sus padres dejaron de transmitir a los estudiantes Sordos fue el desarrollo de sus habilidades sociales e inteligencia emocional ${ }^{16}$; esto desencadenó en vivencias de los estudiantes donde presentaban faltas de conducta, dificultad para seguir normas e instrucciones, carencia de herramientas para hacer ajustes emocionales, entre otros, tal y como lo expresa la docente: "cuando llegaron aquí, miraban con miedo, pateaban las cosas, eran groseros, egoístas, no tenían normas de convivencia" ( $T: E P / R e: A / P: D / G: F / E / R: 22)^{17}$; "de pronto se impacientaba y comenzaba a gritar y a pegarse contra la

${ }^{16}$ Referida a "La capacidad del individuo para identificar su propio estado emocional y gestionarlo de forma adecuada. Esta habilidad repercute de forma muy positiva sobre las personas que la poseen, pues les permite entender y controlar sus impulsos, facilitando las relaciones comunicativas con los demás" (Goleman, 1999:52).

${ }^{17}$ Este código pertenece al procesamiento de análisis respectivo de la información recolectada de las entrevistas realizadas a la docente del ABS: T: Técnica; EP: entrevista a profundidad; R: registro; A: audio; P: participante; D: docente; G: género; F: femenino; R: relato. 
pared" (T:EP/Re:A/P:D/G:F/E/R:11); "era como estar con niños de cuatro años" (T:EP/Re:A/P:D/G:F/E/R:27). Los estudiantes Sordos estaban lejos de las pautas adecuadas de crianza y los estilos educativos que utilizaban los padres no eran los más adecuados; esto empeoró el cuadro formativo de los menores.

Los mismos estudiantes Sordos hoy recuerdan con pena la manera como se relacionaban con los oyentes antes de acceder a la lengua de señas: "recuerdo que me llenaba de rabia y les hacía cosas malas" (T:EP/Re:V/P:ES4/G:M/R12); "es como algo que uno no puede controlar y a todo momento quiere pelear" (T:EP/Re:V/P:ES3/G:F/R22). $\mathrm{Al}$ no poderse comunicar con nadie, los estudiantes se llenaban de resentimiento y rabia, manifestados en expresiones de agresividad con los demás; en el fondo, vivenciaban impotencia y frustración al sentirse rechazados y señalados por los demás. Los padres de familia no sabían lidiar con este asunto.

Respecto a esta cuestión familiar, los estudiantes Sordos tuvieron experiencias angustiosas en cuanto al aspecto clínico: muchas terapias rehabilitadoras y diagnósticos para acceder a dispositivos tecnológi$\cos ^{18}$, como lo expresan los padres: "no perdía la esperanza que mi hijo lograra hablar" (T:EP/Re:A/G:F/P:P1/R:15) ${ }^{19}$; "en la casa también teníamos que hacer muchos ejercicios para que le saliera la voz" (T:EP/ Re:A/G:F/P:P2/R:9); "uno hace lo que los médicos le dicen... es como ese desconocimiento" (T:EP/Re:A/G:F/P:P3/R:21). En estos procedimientos médicos, muchos tiempos familiares fueron arrebatados, dejando a un lado otros tiempos de vida familiar realmente significativos para descubrir el mundo, socializar, jugar, descubrir habilidades;

18 "Cualquier instrumento, equipo o sistema técnico usado por una persona con discapacidad, fabricado especialmente o disponible en el mercado, para prevenir, compensar, mitigar o neutralizar la deficiencia, discapacidad o minusvalía” (Ferrer, 2000, p.23).

${ }^{19}$ Este código pertenece al procesamiento de análisis respectivo de la información recolectada de las entrevistas realizadas a los padres de familia del ABS: T: Técnica; EP: Entrevista a Profundidad; R: registro; A: audio; P: participante; P: padre de familia; G: género; F: femenino; R: relato. 
todos estos sueños dejados atrás por cumplir el anhelo de llegar a ser como la mayoría (Sacks, 2003).

De este modo, los estudiantes Sordos sentían actitudes de rechazo en sus familias y también presenciaban acciones de preferencia con sus hermanos. Las experiencias más marcadas a nivel familiar eran de aislamiento, nula participación en la toma de decisiones de la familia, impotencia por no poder comunicar lo que se siente y se piensa: "quería participar cuando mi familia se reunía a conversar" (T:EP/ $R e: V / P: E S 4 / G: M / R: 9)$; "a mi papá no le interesaba lo que yo pensaba” (T:EP/Re:V/P:ES1/G:F/R:8); "tenía como ese sueño de poder hablar con mi mamá” (T:EP/Re:V/P:ES2/G:F/R:14). Por su parte, los padres también manifiestan que sentían vergüenza de su hijo Sordo: "Yo lo escondía y a mí me daba pena que dijeran que él era sordo" (T:EP/Re:A/P:P4/G:F/R:25); "prefería dejarlo en la casa" (T:EP7/ $R e: A / P: P 5 / G: F / R: 12)$. Todas estas manifestaciones de exclusión generan mayor retraso en el desarrollo comunicativo y de identidad de las personas Sordas (Patiño, Oviedo y Gerner, 2001).

A su vez, los padres sin herramientas para formar a su hijo Sordo viven experiencias dolorosas de lucha y soledad que experimentan con la sociedad, reflejadas en actitudes de discriminación, rechazo, miradas compasivas, desinterés, indiferencia: "muchas veces las personas nos miran, como diciendo "pobrecitos”" (T:EP/Re:A/G:F/P:P2/R:5); "a veces se daba uno cuenta que las personas murmuraban y reparaban al niño" (T:EP/Re:A/G:F/P:P3/R:20); igualmente, en términos educativos, también vivieron experiencias amargas como: "fueron muchos los rechazos que recibimos" (T:EP/Re:A/G:F/P:P4/R:8); "en muchos colegios no me lo recibieron por ser Sordo” (T:EP/Re:A/G:F/P:P5/R:13). Ahora bien, en los colegios donde los recibieron no contaban con las condiciones para atender al hijo Sordo: "era tratado como si escuchara" (T:EP/Re:A/G:F/P:P3/R:16); "tuve muchos problemas con compañeritos" (T:EP/Re:A/G:F/P:P2/R:28). Las familias, entonces, vivieron también momentos de desesperación y desesperanza que los llevó a seguir ciegos el proceso formativo de su hijo Sordo (Vesga \& Vesga, 2015). 
Estas situaciones generaron brechas afectivas entre hijos y padres, pues el problema se profundiza al no haber un puente comunicativo asertivo entre lengua y pensamiento; sumado a esto, se hallan las actitudes de impotencia y desesperación de las familias, las cuales conllevan la agresividad o acciones bruscas que afectan el desarrollo de los propios Sordos (Sacks, 2003). Por ende, la esencia de la identidad del sordo radica tanto en la aceptación y el uso apropiado de la lengua de señas, como en una identificación profunda con los valores y comportamientos en las comunidades de sordos. La familia se convierte en la puerta grande, y la lengua de señas se vuelve la llave a ese mundo de oportunidades y de reconocimiento como personas capaces, hábiles, útiles, dentro de una sociedad diversa (Patiño, Oviedo y Gerner, 2001).

Finalmente, se visibiliza cómo los estudiantes Sordos se encontraron atrapados en el silencio, sin poder expresar sentimientos o pensamientos, sin lograr llegar a procesos de comunicación que los llevara a participar en diálogos con sus padres o amigos, condenados a percibir el mundo a través de su corporalidad y su visión, pero sin recibir alguna retroalimentación al respecto para poder construir y entender nuevos mundos y llegar a crear y transformar el suyo propio. Era necesario brindarles a los estudiantes Sordos otras experiencias donde esta realidad virara a su favor y compensar un poco las huellas de lo que ya habían vivido.

\section{Experiencias: descubro la voz en mis manos}

En el desarrollo de esta categoría se presentan las experiencias de los estudiantes Sordos frente a las transformaciones vividas en la adquisición de su primera lengua tardía (Lengua de Señas Colombiana) durante cuatro años de escolaridad en el ABS de Popayán.

Las primeras experiencias de los estudiantes Sordos en la adquisición de su Lengua estuvieron mediadas por sentimientos de miedo, asombro, incertidumbre y extrañeza; ellos nunca habían tenido contacto con dicha lengua: "me empezaron a hablar y yo no entendía absolutamente nada" (T:EP/Re:V/P:ES2/G:M/R16); "Me daba como pena, como inseguridad, como susto" (T:EP/Re:V/P:ES4/G:M/R10). Poco a 
poco, los estudiantes Sordos se sumergieron en procesos de interacción comunicativa donde, a través de sus manos, descubrieron que podían expresar y comunicar: "me sentí muy feliz cuando me hice entender de los demás” (T:EP/Re:V/P:ES1/G:F/R17); “nunca pensé que mis manos fueran mi voz" (T:EP/Re:V/P:ES2/G:M/R8). Los Sordos avanzan en sus habilidades comunicativas y, a la par, vivencian las ventajas y funciones de una lengua viso-gestual (Cortés \&y Barreto, 2012).

Para la generación de experiencias educativas en la enseñanza de una primera lengua, la docente del aula reflexiona y reconfigura sus prácticas pedagógicas adaptándolas a las necesidades de los Sordos; en este sentido, la docente expresa: "al comienzo, uno se siente impotente; los Sordos necesitan de estrategias distintas a los oyentes, allí está el reto" (T:EP/Re:A/P:D/G:F/R25); “después entendí que uno como maestro debe valerse de cualquier medio para llegar a esas diferencias; en este caso, la auditiva; y allí es donde hay que ser muy persistentes y creativos" (T:EP/Re:A/P:D/G:F/R4). Es así como la experiencia del docente avanza respetando las diferencias y los ritmos de cada estudiante Sordo: "es imposible pretender tratar a todos por igual, hay que llegar a la personalización de las necesidades de cada uno para, desde allí, potenciar sus capacidades" (T:EP/Re:A/P:D/G:F/R14); hay que sumergirse, entonces, en la historia genética, biológica, familiar y social de cada estudiante para guiar procesos reales de formación (Padden y Humphries, 2005).

En este sentido, los estudiantes Sordos experimentan cómo su aula y su escuela se transforman con el propósito de crear ambientes de aprendizaje realmente inclusivos (Aguilar et al, 2008); por ejemplo, la docente manifiesta: "se gestionaron recursos para comprar material didáctico y así apoyar todo el proceso pedagógico” (T:EP/Re:A/P:D/ $\mathrm{G}: \mathrm{F} / \mathrm{R} 32)$; "me capacité en la formulación y desarrollo de proyectos de aula que posibilitó generar ambientes de aprendizaje para la adquisición de la lengua y demás conocimientos de las áreas" (T:EP/Re:A/P:D/G:F/R19). En consecuencia, los estudiantes Sordos expresan con alegría y satisfacción cómo la escuela, específicamente lo que acontece 
en el aula, se fue adaptando a ellos y hoy la sienten como el lugar que les posibilitó "volver a nacer".

Es de esta manera como los estudiantes Sordos, a través de su input visual, vivenciaron no solo avances en su proceso de comunicación e interacción, sino en aprendizajes que para ellos fueron como un despertar: "ahora, si no entiendo algo, puedo preguntar... es como esa tranquilidad de poder acceder a información que antes no me llegaba" (T:EP/Re:V/P:ES3/G:F/R20); “yo pienso que he aprendido mucho, sé de cosas peligrosas como las drogas o la violencia sexual, que antes no lo sabía” (T:EP/Re:V/P:ES5/G:F/R26)". Los estudiantes sienten cómo la lengua de señas se convierte, además, en mediación del pensamiento y acceso al conocimiento del mundo (González, 2009).

Otra de las experiencias de los estudiantes consiste en la manera como acceden a procesos de reflexión de sus propias acciones; esto conduce a transformaciones de sus conductas y comportamientos negativos: "los niños han mostrado cambios en la forma de relacionarse con los demás, ya se comportan con respeto y responsabilidad en muchos de sus actos" (T:EP/Re:V/P:D/G:F/R10); "Los Sordos también aprenden las habilidades sociales, pero hay que escucharlos y llevarlos a reflexionar frente a su responsabilidad social" (T:EP/Re:V/P:D/G:F/ R18). En el aula, a partir de diálogos en grupo, se vivencian procesos de solución de conflictos, donde los Sordos aprenden a desarrollar habilidades emocionales y sociales; esto los ha llevado a fortalecer su autoestima y la confianza en sí mismos (Carter y Mireles, 2016).

Así pues, en la escuela, los estudiantes Sordos avanzan hacia experiencias positivas y gratas de amistad: "yo me siento muy feliz con mis amigos; compartimos, jugamos, también a veces peleamos, pero siempre nos disculpamos" (T:EP/Re:V/P:ES4/G:M/R:11); “a veces que estoy triste mis amigos me apoyan y se preocupan por mí, eso me hace sentir bien" (T:EP/Re:V/P:ES2/G:F/R:17). También manifiestan, a veces, tener peleas y conflictos que en principio se asumían como fracasos, adoptando actitudes negativas hacia ellos mismos y hacia los demás; esto llevó a la necesidad de generar espacios formativos para la resiliencia, 
siendo esta esa capacidad de adaptarse y superar las adversidades, asumidas estas como una oportunidad de superación (Manciaux, 2003).

Con el propósito de elevar su autoestima y los procesos de autoconocimiento, los estudiantes indagaron sobre su historia familiar, el origen de su sordera, la vida de otros sordos, sus posibilidades y limitaciones: "sé por qué nací Sordo y que uso una lengua distinta a los demás, y esa es mi forma de comunicación" (T:EP//Re:V/P:ES5/G:F/R19); "pensaba que yo era el único Sordo en el mundo, pero no, hay muchos en el mundo y no es un impedimento para salir adelante" (T:EP//Re:V/P:ES2/G:M/R6). Estas vivencias en el aula, donde se toca la cultura Sorda, llevó poco a poco a los estudiantes a reconfigurar sus procesos de identidad que, según Sacks (2003), se hace a través de un lenguaje interior de cada persona, incidiendo en la identidad real de cada sujeto, posibilitando dar sentido, elaborar significados y conceptos, y desde allí construir un mundo propio.

De este modo, los estudiantes empiezan a sentir pertenencia a la cultura Sorda: "me gusta saber qué hacen otros Sordos de Colombia y el mundo" (T:EP//Re:V/P:ES3/G:F/R25); "a mí me encanta cuando otros Sordos adultos vienen a visitarnos y nos cuentan sus historias de vida” (T:EP// Re:V/P:ES4/G:F/R14). Las fortalezas de trabajar por proyectos pedagógicos biculturales ha permitido que los Sordos fortalezcan sus procesos de identidad y se sientan orgullosos de pertenecer a dos culturas.

En la historia, la cultura Sorda siempre fue considerada inferior a la de los oyentes; los estudiantes Sordos han ido cambiando esta concepción, pues gracias al desarrollo de sus capacidades y habilidades se han destacado en el ámbito escolar, incluso de mejor manera que los mismos oyentes: "Los demás niños quedan con la boca abierta cuando nos ven bailar" (T:EP/Re:V/P:ES3/G:F/R:6); "hemos ganado varios [juegos] interclases y no nos da miedo salir a participar en actos culturales"(T:EP/Re:V/P:ES2/G:M/R:15). Las experiencias que los estudiantes narran sobre su participación en salidas pedagógicas, actividades deportivas, culturales, académicas, les ha llevado a destacarse ente los demás por sus capacidades y habilidades; aspectos formativos que 
permiten que, en la comunidad educativa, se cambie la concepción de 'pobrecitos' por una de admiración y valoración.

En este sentido, los estudiantes Sordos han sentido el respeto y la admiración de la comunidad educativa: "siempre que hacemos algo, nos sentimos muy bien, pues todos en la escuela nos felicitan por lo que hacemos" (T:EP/Re:V/P:ES2/G:F/R:6); "ahora me siento capaz de hacer muchas cosas, a veces nosotros les enseñamos a los niños oyentes, si ellos no saben" (T:EP/Re:V/P:ES3/G:F/R:23). Al interactuar con los estudiantes se refleja un orgullo entre ellos y, al mismo tiempo, alegría y satisfacción al recordar las experiencias escondidas en las filmaciones de actividades artísticas donde ellos aparecen, las cuales, a lo largo de cuatro años, han podido preparar y presentar en público ante toda la comunidad educativa; de acuerdo con esto, se podría decir que los procesos de inclusión reales son los que resultan del verdadero trabajo con las comunidades, su empoderamiento y visibilización (González, 2011).

Por consiguiente, a través de estas experiencias los estudiantes descubren sus propias habilidades y empiezan a tomar decisiones frente a su futuro: "he aprendido que practicando y con esfuerzo puedo aprender y alcanzar lo que quiero ser" (T:EP/Re:V/P:ES4/G:F/R:18); "Yo quiero seguir aprendiendo más, para poder estudiar y ser más adelante una profesional" (T:EP/Re:V/P:ES1/G:F/R:19). Estas visiones esperanzadoras de futuro motivan a los niños a un mayor compromiso con las actividades escolares: "a mí me gusta venir aquí, aprender, hacer los trabajos para poder seguir adelante” (T:EP/Re:V/P:ES43/G:M/R:13). La inclusión educativa también es, entonces, empezar a proyectar a los estudiantes a una vida profesional basada en la diversidad desde la diferencia como potencialidad (Padilla, 2011).

Igualmente, las experiencias escolares vividas por los estudiantes Sordos se han extendido a sus entornos familiares: " yo le digo a mi mamá que no me hable, que soy Sorda, que nos comuniquemos por la lengua de señas" (T:EP/Re:V/P:ES3/G:F/R:27); "ahora exijo en mi casa que me tengan en cuenta y que me informen de lo que allí pasa” 
(T:EP/Re:V/P:ES2/G:M/R:23). Los padres de familia han transformado la concepción que tenían de la sordera, de la lengua de señas y de la cultura Sorda: "ahora he aprendido la lengua y me puedo comunicar con mi hija... se siente muy bien" (T:EP/Re:A/P:P4/G:F/R:16); "ahora para mí es un orgullo y trato de formar a las personas ignorantes en el tema” (T:EP/Re:A/P:P1/G:F/R:27). Las familias se han ido introduciendo en la lengua de señas y han avanzado en procesos comunicativos con los estudiantes Sordos; este proceso es fundamental para la vida del niño, pues la familia es la primera que posibilita los procesos de inclusión (Verdugo y Rodríguez, 2008)

Es así como estas experiencias en la adquisición de la lengua de señas con los estudiantes Sordos les posibilitó no solo comprender el mundo sino poder crear otros: "Ahora puedo entender qué pasa aquí, en mi casa, con mis amigos; puedo expresar lo que quiero hacer, crear, pintar, actuar" (T:EP/Re:V/P:ES2/G:M/R:31). De esta manera, como lo afirma Sacks (2003), gracias al lenguaje los niños lograron acceder al campo puramente simbólico de pasado y futuro, de lugares remotos, de relaciones ideales, de metáforas, de creaciones posibles e imposibles en interacción con su mundo, con los otros y con su entorno.

\section{Conclusiones}

Esta experiencia del ABS, acontecida en Popayán, es un ejemplo de que sí es posible brindar una educación de calidad a estudiantes Sordos y que, a través de esta experiencia, se logró transformar la realidad de los niños y adolescentes Sordos y sus familias, mostrando un camino para garantizar reales procesos de inclusión educativa, brindándoles espacios de participación y oportunidades, para que ellos construyan su mundo y, a partir de allí, generen posibilidades de contribución a una sociedad diversa.

Se requiere empezar a trabajar desde distintos sectores sociales por una educación inclusiva para estudiantes sordos donde se generen ambientes de aprendizaje para el desarrollo de su primera lengua (la lengua de señas), basados en procesos de interacción con toda la 
comunidad educativa y con su entorno familiar, donde avancen en el desarrollo de sus habilidades cognitivas, motrices, emocionales y para la vida; solo así se garantizará que los estudiantes Sordos puedan tener una educación de calidad.

Es de vital importancia continuar avanzando en procesos de sensibilización a la comunidad en general frente a la Sordera, la lengua de señas, la cultura Sorda, para que se sigan transformando concepciones erróneas que permanecen en la sociedad hoy en día y que siguen generando barreras para esta población, para así avanzar hacia la construcción de una cultura inclusiva con más ímpetu en las ciudades periféricas de Colombia.

Es urgente vincular a entidades nacionales y gubernamentales como el ICBF y el programa DeCero a Siempre en procesos de reflexión frente a la educación de los Sordos, para que desde sus acciones hagan cumplir la política pública de inclusión para la infancia Sorda y así garantizar a temprana edad que los niños accedan a su lengua de señas y sean atendidos no solo por el sector salud sino también a partir de sus necesidades pedagógicas (Castro, 2003).

Es necesario que la población civil conozca las políticas públicas frente a la educación de los Sordos y que entre todos se haga veeduría ciudadana en su cumplimiento en todo el territorio nacional para garantizar una educación de calidad a infantes y jóvenes sordos, así como la garantía del acompañamiento de los profesionales de apoyo, necesarios para la educación de los Sordos como son docentes usuarios de Lengua de Señas Colombiana, modelos lingüísticos e intérpretes de acuerdo al nivel educativo donde se encuentre la persona Sorda. 


\section{Referencias}

Ayala, R. (2008). La metodología fenomenológico hermenéutica de M. Van manen en el campo de la investigación educativa. Posibilidades y primeras experiencias. Revista de Investigación Educativa, 26(2), 409-430. Recuperado de http:// www.redalyc.org/pdf/2833/283321909008.pdf

Batthyány, K., Cabrera, M. (2011). Metodología de la investigación en Ciencias Sociales. Apuntes para un curso inicial. Montevideo: Universidad de la República. Recuperado de http://www.cse.edu.uy/sites/www.cse.edu.uy/files/ documentos/FCS Batthianny 2011-07-27-lowres.pdf

Bolívar, A. (2012). Justicia social y equidad escolar. Una revisión actual. Revista internacional de educación para la justicia social, 1(1), 9-45. Recuperado de http://www.rinace.net/riejs/numeros/vol1-num1/art1.pdf

Cabezas, R. (2014). Compartiendo algunas reflexiones sobre la inclusión educativa de personas sordas. Recuperado de: http://www.culturasorda.eu/resources/ Cabezas Reflexiones inclusion educativa personas sordas 2014.pdf

Carter, M. J., Mireles, D. C. (2016). Exploring the Relationship Between Deaf Identity Verification Processes and Self-Esteem. Identity, 16(2), 102-114. Recuperado de http://www.tandfonline.com/doi/abs/10.1080/15283488.2016.1159963?needAccess $=$ true

Castro, P. (2003). Aprendizaje del lenguaje en niños sordos: fundamentos para la adquisición temprana del lenguaje de señas. Psicología Científica, 5(10), 15-27. Recuperado de: http://www.psicologiacientifica.com/lenguaje-de-senas-aprendizaje

Cortés, Y., Barreto, A. (2012). Variación sociolingüística en la LSC. Diplomado en Planeación Lingüística. Bogotá: Universidad Nacional de Colombia.

Denzin, N., K., Lincoln, Y., S. (2005). The Sage Handbook of Qualitative Research. London: Sage.

Domínguez, A. (2009). Educación para la inclusión de alumnos sordos. Revista Latinoamericana de Educación Inclusiva, 3(1), 45-61. Recuperado de: http:// 
www.rinace.net/rlei/numeros/vol3-num1/art4.pdf.

Ferrer, A. (2000). Ayudas técnicas y tecnológicas para la vida cotidiana. En Rasgos Sociológicos y Culturales de las Personas Sordas. Una aproximación a la situación del colectivo de personas sordas en la Comunidad Valenciana. Valencia: Fundación FESORD-CV.

García, M., Ibañez, J., Alvira, F. (2005). El análisis de la realidad social. Métodos y técnicas de investigación social. Madrid: Alianza.

Goleman, D. (1999). La práctica de la inteligencia emocional. Barcelona: Kairós.

González, V. (2011). Un acercamiento histórico a la Comunidad sorda de Bogotá. Bogotá: Fenascol. Recuperado de: http://www.fenascol.org.co/SEDasignaturaLSC/doctos/HISTORIA2.pdf

González, F. (2009). Claves para la educación de los/las niños-niñas. Valencia: Serrano Villalba, Cristóbal.

Larrosa, J. (2006). Sobre la experiencia. Revista de Psicología, 19, 87-112. Recuperado de: http://www.raco.cat/index.php/Aloma/article/viewFile/103367/154553

Lissi, M., Svartholm, K., González, M. (2012). El Enfoque Bilingüe en la Educación de Sordos: sus implicancias para la enseñanza y aprendizaje de la lengua escrita. Estudios Pedagógicos, XXXVIII (2), 299-320. Recuperado de: http://www. scielo.cl/pdf/estped/v38n2/art19.pdf

Manciaux, M.(2003). La resiliencia : resistir y rehacerse. Barcelona: Gedisa

Marines, M., Heredia, N., Solis, L., Mena, D. (2014). Taller Multidisciplinario para el Desarrollo de Competencias de Comunicación Lingüística de la Investigación. Taller Multidisciplinario, 7(2), 41-50. doi: 10.4067/So71850062014000200006

Martínez, J. (2004). Estrategias metodológicas y técnicas para la investigación social. México: Universidad Mesoamericana. Recuperado de: $\underline{\text { http://www. }}$ geiuma-oax.net/sam/estrategiasmetytecnicas.pdf 
Medina, E. (2005). Representaciones y políticas de la educación sobre los Sordos y la sordera en Colombia. Revista en educación y pedagogía, 17, 71-81. Recuperado de: http://www.cultura-sorda.eu/resources/Medina Moneada Representaciones politicas educacion sordos Colombia 2005.pdf

Ministerio de Educación Nacional. (2005). Lineamientos de política para la atención educativa a poblaciones vulnerables. Bogotá: MEN. Recuperado de: http:// www.mineducacion.gov.co/cvn/1665/article-90668.html

Monsalve, A., Núñez, F. (2016). La importancia del diagnóstico e intervención temprana para el desarrollo de los niños sordos: Los programas de detección precoz de la hipoacusia. Intervención Psicosocial, 15(1), 7-28. Recuperado de: http://scielo.isciii.es/scielo.php?script=sci arttext\&pi$\underline{\mathrm{d}=\mathrm{S} 1132-05592006000100002 \& \operatorname{lng}=\mathrm{es}}$.

Murcia, N., Jaramillo, L. (2009). Investigación cualitativa. La complementariedad etnográfica. Armenia: Kinesis.

Niño, A., Velasco, V., Toro, C. (2005). El borde como espacio articulador de la ciudad actual y su entorno. Revista Ingenierías Universidad de Medellín, 4(7) 55-65. Recuperado de http://www3.redalyc.org/articulo.oa?id=75004705

Orozco, Z. (2014). Experiencias de reconocimiento y menosprecio en la atención de personas sordas en escenarios educativos. Medellín: Cinde, Universidad de Manizales. Recuperado de: http://ridum.umanizales.edu.co:8080/xmlui/ handle/6789/1230

Oviedo, A. (2006). La visión de personas oyentes sobre la cultura sorda. El Congreso de Milán, 1880. Recuperado de: http://www.cultura-sorda.eu.

Padden, C., Humphries, T. (2005). Inside Deaf Culture. Cambrigde: Harvard University Press.

Padilla, A. (2011). Inclusión educativa de personas con discapacidad. Revista Colombiana de Psiquiatría, 40(4), 670-699. Recuperado de: http://www.redalyc. org/articulo.oa?id=80622345006 
Patiño, L. Oviedo, A., Gerner, B. (Eds.) (2001). El estilo sordo: ensayos sobre comunidades y culturas de las personas sordas en Iberoamérica. Volumen 2. Santiago de Cali: Universidad del Valle.

Rey, M. (2008). El cuerpo en la construcción de la identidad de los sordos. Papeles de trabajo - Centro de Estudios Interdisciplinarios en Etnolingüística y Antropología Socio-Cultural, (16). Recuperado de: http://www.scielo.org.ar/scielo. php?script=sci_arttext\&pid=S1852-45082008000100005\&lng=es\&tlng=es.

Robles, M. (2012). Tendencia educativa bilingüe y bicultural para la educación del sordo un nuevo camino hacia la inclusión. Unirevista, (1), 78-86. Recuperado

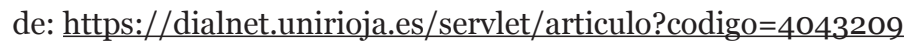

Sacks, O. (2003).Veo una voz. Barcelona: Editorial Anagrama.

Verdugo, M., Rodríguez, A. (2008). Valoración de la inclusión educativa desde diferentes perspectivas. Siglo Cero, 39(4), 5-25. Recuperado de: http://www. revistaeducacion.mec.es/re349/re349 02.pdf

Vesga, L. \& Vesga, J. (2015). Una exclusión que se perpetúa: tensiones entre docentes, niños sordos y niños oyentes en escenarios escolares de Popayán. Revista Virtual Universidad Católica del Norte, 46, 115-128. Recuperado de: http:// revistavirtual.ucn.edu.co/index.php/RevistaUCN/article/viewFile/703/1230 